\title{
Mis aportaciones como hidrometeorólogo
}

\author{
Josep Pascual i Massaguer*
}

Santa Anna, 49, L'Estartit, 17258 Girona.

* Corresponding author: jpascual@meteolestartit.cat

Received: 11/06/19 Accepted: 03/10/19

\section{RESUMEN}

Mis aportaciones como hidrometeorólogo

Se describen aquí las actividades de Josep Pascual i Massaguer como hidrometeorólogo, el cual comenzó a registrar en 1968 de manera privada, primero, y pública (más tarde) numerosas observaciones meteorológicas, térmicas e hidrodinámicas en el medio marino y el dulceacuícola del entorno de Torroella de Montgrí (NE España), incluyendo las islas Medas, el mar Mediterráneo, el lago de Banyoles, el río Ter y numerosas fuentes. Sus series de datos térmicos y del nivel marino no solo se encuentran entre las más largas de la Península Ibérica, sino que su frecuencia de registro resulta también muy notable. Ambos aspectos convierten a sus series de datos en especialmente útiles para el estudio de los efectos del calentamiento global en nuestro territorio. Además del enorme número de variables que registra a distintas escalas temporales y espaciales, el señor Pascual i Massaguer ha tenido tiempo de dedicarse también a otras actividades culturales, como la música folklórica del NE peninsular.

Palabras clave: series de datos a largo plazo, meteorología, temperaturas atmosférica y acuática, nivel del agua en ecosistemas acuáticos, mares, lagos, ríos, sardanas

\section{ABSTRACT}

\section{My contributions as hydrometeorologist}

The scientific activities of Mr Josep Pascual i Massaguer as hydrometeorologist are reported here. He started his meteorological, marine and limnological observations privately in 1968, but later they were partially supported by public funding. His long-term records were undertaken in the marine and freshwater surroundings of Torroella de Montgri (NE Spain), including Medes Islands, Mediterranean sea, Banyoles lake, Ter river and several springs. The data series that he is recording are among the longest and more frequent ones in the Iberian Peninsule. Both features make his records very useful to study global warming effects in that area. In addition to the huge number of variables recorded, this hydrometeorologist is also able to devote his endeavours to cultural issues, such as folk music of NE Spain.

Key words: long-term data series, meteorology, air and water temperatures, water level, ocean, lakes, streams, sardana music 


\section{INTRODUCCIÓN}

\section{Mis comienzos}

Nací en 1950 en l'Estartit, un pequeño pueblo de la costa de Girona. Durante mi infancia, era básicamente un lugar de pescadores y payeses. Todavía el turismo -que es ahora, la primera fuente de ocupación- era muy incipiente. Mi familia, por parte de mi padre (Rafel), venía de pescadores y por parte de mi madre (Esperança), de payeses. En aquella época la pesca era la principal ocupación de las familias en el pueblo. También había entonces algunas tiendas de ultramarinos, un par de carnicerías, tres hornos de pan, algunos herreros, un carpintero.... y dos mestres d'aixa, o astilleros de fabricación de las barcas de madera de la época, las cuales se harían años más tarde con fibra de vidrio. El pueblo no llegaba a los 1000 habitantes.

De muy pequeño, mi padre y mi abuelo paterno también llamado Rafel ("Felet" para la familia y amigos) me llevaban a pescar en su barca (Fig. 1). La pesca era artesanal, pues se hacía con redes, palangres, nasas, e incluso había en el puerto seis barcas de teranyina (redes de cerco) para la pesca de sardina y anchoa. Todas estas embarcaciones carecían aún de las máquinas que ayudan a sacar las redes del mar y, por ello, se tenían que extraer a mano. A causa del esfuerzo que suponía, eso reducía el número de redes que se echaban al mar y, sobre todo, la profundidad máxima a la que se podían situar, porque el esfuerzo humano aumentaba con la profundidad debido al peso de la misma red, al de las capturas y a las corrientes. Todo esto, que ahora nos podría parecer un inconveniente, en realidad hacía la pesca sostenible, y hacía posible que hubiese zonas del mar donde los peces se podían reproducir libremente, pues no se pescaba en ellas.

El tipo más habitual de pesca consistía en dejar en el mar los aparejos un día y sacarlos al siguiente con la pesca que hubiesen capturado, pues los peces siguen la luz y normalmente se enredan en las redes al amanecer y al atardecer, con las primeras y últimas horas del día. Esto, presuponía que, en una época en que los modelos numéricos de previsión no existían, el mismo pescador debía hacer sus propias predicciones
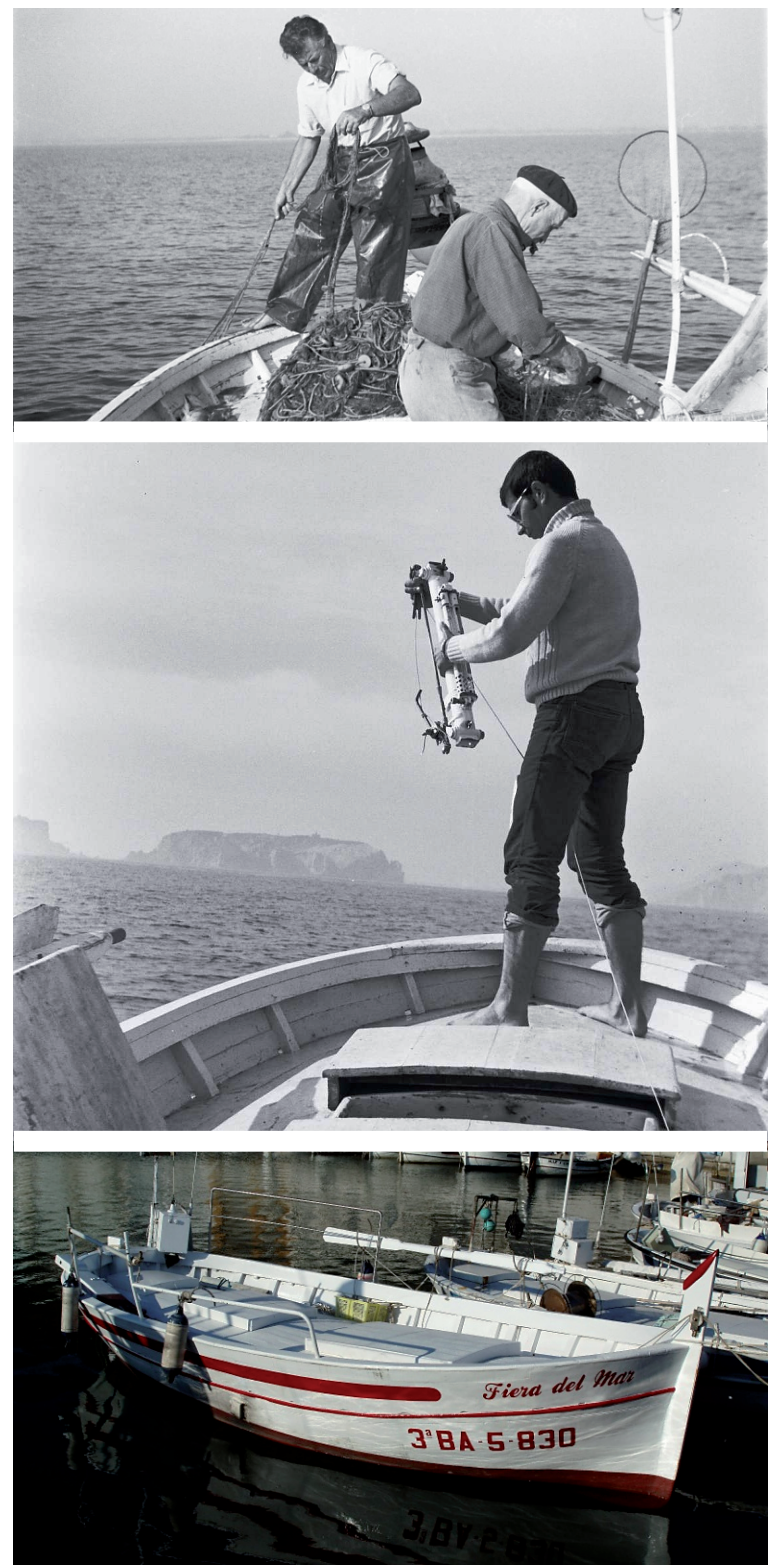

Figura 1. Tres instantáneas de la barca de mi abuelo, construida en 1946 con la que continúo saliendo a realizar mis observaciones marinas. En la fotografía superior, mi padre (a la izquierda) y mi abuelo, faenando en ella con trasmallo en 1970. En la foto intermedia, un servidor en acción en 1975 con una botella Nansen que lleva dos termómetros de inversión adosados. En la inferior, la barca en la actualidad, restaurada en 2009. The boat of my grandfather, built in 1946, which I still sail to record my oceanographical observations. In the upper photograph my father (on the left) and my grandfather (on the right) are preparing to throw fishing nets in 1970. The middle picture shows me in action in 1975 with a Nansen bottle and two attached inverse thermometers. In the lower photograph, the current boat which I use for my observations is shown whose name reads as "The Sea Beast" and was restored in 2009. 
empíricas tanto sobre el tiempo atmosférico local como sobre el estado de la mar. Las conversaciones entre mi padre y mi abuelo hablando del posible tiempo del día siguiente que condicionaba el mejor lugar de la costa para dejar las redes quedaban grabadas en mi mente, y eso fue el origen de mi afición a la meteorología y a la oceanografía física.

A los 13 años ya tomaba apuntes del tiempo, pese a que carecía de aparatos de medida. A los 15 , con un termómetro de 20 pesetas [algo menos de 16 céntimos de euro al cambio actual, si no tenemos en cuenta la inflación] que me regalaron mis padres, un barómetro aneroide que nos regalaron unos amigos alemanes (de los primeros turistas que llegaron al pueblo) y con un bote de conserva como pluviómetro empecé a tomar datos de temperatura, presión atmosférica, lluvia $\mathrm{y}$, de manera visual, a reconocer el estado del cielo y los vientos.

Mi relación con el mar y la pesca me motivó la curiosidad sobre la temperatura que debía tener el mar en cada momento. Notaba que los pescadores tenían un conocimiento empírico del clima local muy útil para su trabajo, pero yo quería darle un fundamento científico. Durante unos meses, tomé datos de temperatura del mar en la playa (1967-1968). Un par de años más tarde, cuando ya disponía de un termómetro de máxima y mínima, quise saber qué temperatura tenía el mar por debajo de la superficie. Al final, conseguí que un día de agosto de 1968 mi padre me dejara la barca (yo no tenía entonces ninguna titulación de patrón) para salir a medio kilómetro del puerto y dejar el termómetro de máxima y mínima a diferentes profundidades, hasta los 20 metros, lo cual me permitió comprobar que en verano la temperatura del agua era más baja a mayor profundidad. $\mathrm{O}$ sea, que a los 18 años, sin ayuda de nadie, descubrí la estratificación térmica.

Acabado el bachillerato, fui a estudiar ingeniería técnica agrícola en la Escuela de Barcelona. Por aquel entonces, había en el pueblo dos topógrafos que habían trabajado para el Catastro (los señores Marcel·lí Audivert y Juan Pujadas), entonces ya jubilados, que a veces me invitaban a ver sus trabajos, sus planos y sus aparatos de medida. Esto me atrajo a la Topografía, ya que también me gustaban las cartas náuticas. Al no tener cerca escuelas de esa especialidad, estudié en la Escuela de Agricultura de Barcelona, donde también había asignaturas de topografía y dibujo.

Una de las primeras cosas que hice entonces fue acudir al centro meteorológico de la época (entonces Servicio Meteorológico Nacional) para ver si me podían proporcionar algún aparato. Cuando llegué, la persona que me atendió -el Dr. Tomás Quevedo- estaba rellenando un mapa isobárico con los datos que iba recibiendo por télex para dibujar a lápiz posteriormente las líneas isobáricas. Como crédito, le mostré una gráfica de la temperatura del mar en l'Estartit que había hecho gracias al termómetro de máxima y mínima. Debió gustarle, porque ese mismo día él me dio un pluviómetro que, ahora bastante viejo, sigue funcionando aún en una pequeña estación situada en la montaña del pueblo (Roca Maura), a 220 metros de altura.

La casualidad hizo que, al día siguiente, acudiera a ese mismo centro el Dr. Antoni Ballester, del Instituto de Investigaciones Pesqueras de Barcelona (hoy en día, el Instituto de Ciencias del Mar, CSIC), para solicitar datos meteorológicos pasados, coincidentes con una campaña oceanográfica que se había hecho desde este Instituto. Y a un lado de la misma mesa, seguía la gráfica que yo había llevado el día anterior. Antoni se sorprendió de ver unos datos de temperatura del mar de los cuales no tenía noticia. Enseguida, pidió el número de teléfono de mi casa y me llamó, rogándome que le fuera a ver al Instituto de Pesqueras, situado en la Barceloneta, para tener una conversación.

Allí me presenté, y me pidió que le explicara cómo había tomado los datos, e inmediatamente notó mi afición. El Dr. Ballester era un gran científico y una excelente persona, y me comentó muchas cosas de su Instituto. Me sugirió que, si seguía teniendo interés por el tema marino, me podía dejar una botella Nansen y un termómetro de inversión para tomar los datos térmicos con más precisión. Pero me alertó (era otra época): "te dejo esto, pero "oficialmente" estos instrumentos están en nuestro almacén".

Durante un par de años (1969-1971), tomé datos de temperatura marina de manera irregular, ya que pasaba mucho tiempo en Barcelona con los estudios y después vino la "mili" en Melilla, 
lo cual me impidió tomar datos de dicha temperatura durante más de un año, si bien los datos atmosféricos (básicamente, los de temperatura, lluvia, humedad, presión, estado del cielo, viento, etc.) los tomaban mi madre y mi hermana Carmen en una especie de ficha diaria que yo les había preparado.

\section{Mis aficiones al margen de la hidrometeorología}

Como he comentado antes, cuando yo era pequeño en l'Estartit (como en la mayoría de pueblos de la Costa Brava), el turismo era muy incipiente; se limitaba a algunas familias, básicamente de Barcelona, que venían a pasar unas semanas en verano, aunque algunas de ellas tenían aquí su segunda residencia. Bien es cierto que también empezaban a llegar los primeros extranjeros, ingleses en su mayoría.

En el pueblo no había cine entonces (sí hubo uno con proyecciones de cine mudo antes de la guerra civil). Solamente había música por la fiesta mayor, en verano, por Santa Ana, patrona del pueblo, y también por Nuestra Señora del Carmen, patrona de los pescadores, cuando se llevaba a la Virgen en procesión marinera por la bahía. En estos casos venía una cobla-orquestra, una típica formación musical catalana, que por la mañana acompañaba la misa, después tocaba una sardana en la plaza (Fig. 2), habitualmente otras sardanas por la tarde y, por último, amenizaba un baile (a veces también concierto) por la noche.

Una de las cosas que me encantaba de la formación de cobla, compuesta habitualmente por once instrumentos (diez de viento y un contrabajo), era ver cómo -tocando todos juntos, pero diferentes notas cada uno- había armonía y podían acabar todos los instrumentos al mismo tiempo. Así que, de joven, me apunté en el pueblo vecino de Torroella de Montgrí a un grupo que bailaba sardanas y otras danzas regionales con vestidos típicos de la zona. Tengo un gran recuer-

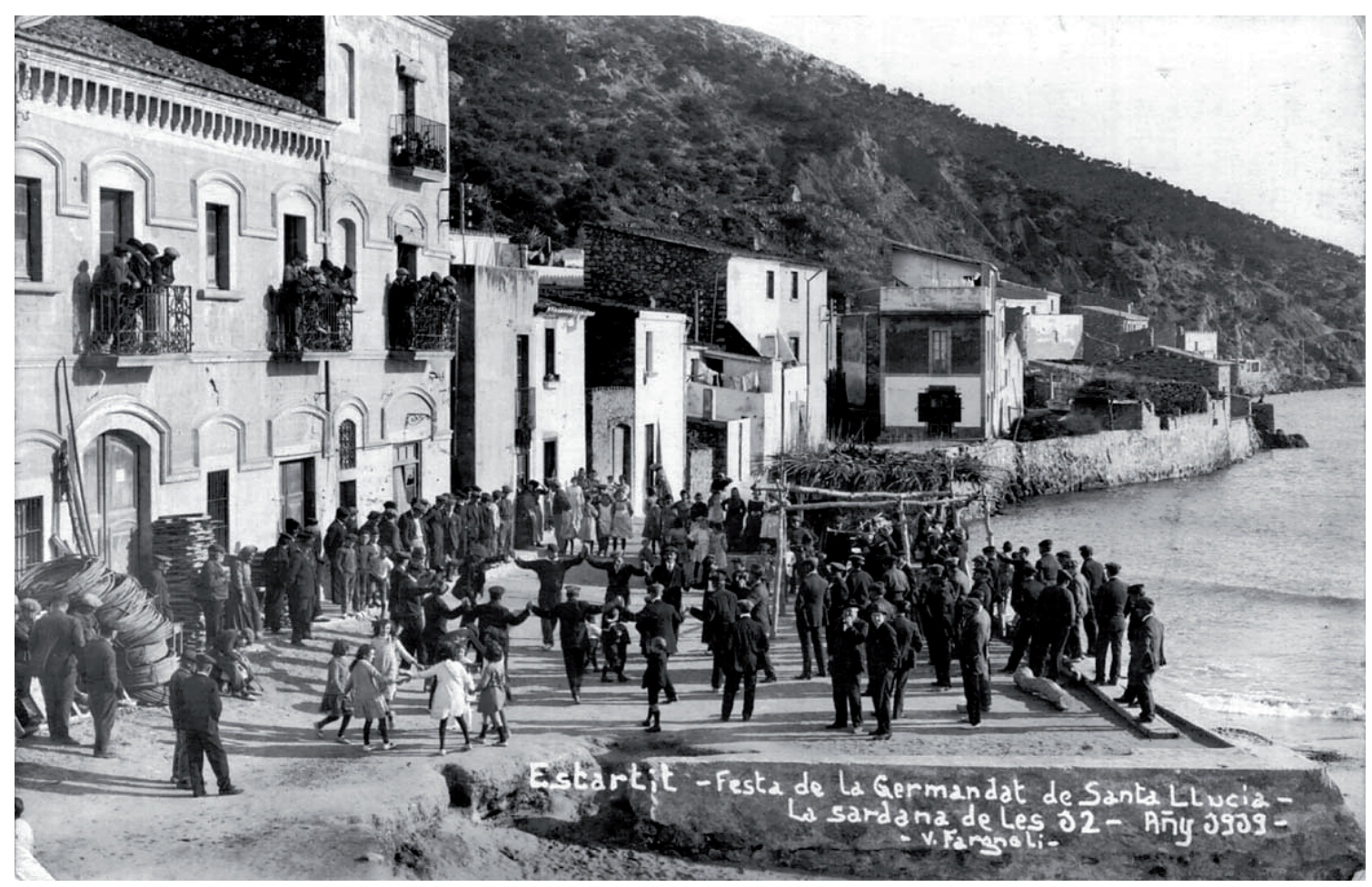

Figura 2. Sardana en l'Estartit hace un siglo, la fiesta de Santa Llúcia, que se celebraba el 13 de Diciembre. Fotografía de Valentín Fargnoli, tomada en 1919. Sardana dance in l'Estartit (Girona) in 1919 to celebrate the town feast (Santa Llúcia) in December, the 13th. Photograph taken by Valentín Fargnoli. 
do de aquellos años. Básicamente actuábamos durante los fines de semana en el pueblo o pueblos vecinos. Pero hicimos también algún viaje más lejos; por ejemplo, estuvimos una semana en Holanda y también unos días en Málaga, donde hubo una demostración de danzas regionales de diferentes puntos de España.

En una de esas salidas, un compañero estudiante de música me dijo: "sabes, me he comprado un fiscorno [instrumento que se utiliza en la cobla para tocar sardanas] de segunda mano". Y se me ocurrió decir: "a mí también me gustaría tener un instrumento de música". Él me contestó: "este señor, que me ha vendido el instrumento, tiene también una tenora [la tenora es otro instrumento de la cobla], y la vende". Y ahí me tienes, a los 27 años, cuando voy a ver a aquel señor, nos entendemos con el precio y le compro la tenora. Yo pensé: "¿y ahora qué hago?"; porque no tenía ni idea de música. Así que me fui a buscar a un profesor para que me diese clases semanales de música. Al cabo de unos cuatro años, me pidieron que entrara a tocar en la agrupación de sardanas donde aún sigo: la Principal de Banyoles. Y vamos por las fiestas mayores de los pueblos de la comarca y comarcas vecinas. Es una actividad muy agradecida, ya que siempre haces amigos. Podéis ver algunas de nuestras actuaciones en la sección de "Enllaços" de https://principaldebanyoles.cat.

También desde hace años estoy en una coral, donde cantamos canciones tradicionales catalanas y de otras partes de España y del Mundo, algunas piezas clásicas o los típicos villancicos en época de Navidad.

Cuando empecé a oficiar como topógrafo, sobre todo hacía trabajos particulares para payeses: mediciones de sus campos para el replanteo de frutales, mediciones de nivelación para riegos, pero también medidas de parcelas para algún arquitecto. Había observado que las cartas náuticas de la zona tenían bastantes de sus topónimos erróneos o mal escritos. Recuerdo que mi abuelo "Felet" me explicaba los nombres de los diferentes rincones de nuestra costa y yo los iba anotando en una libreta. Se me ocurrió que, aprovechando que ya disponía de un teodolito para el trabajo, podía hacer un mapa de la zona a escala y poner los nombres verdaderos en el lugar correcto. A ratos, fui haciéndolo entre 1973 y 1976. Finalmente, el mapa se llegó a publicar en 1977. Tuvo mucho éxito, y en 1986 se imprimió otra edición. Lo llamé "Carta (náutica) de l'Estartit".

\section{MIS MÉTODOS Y OBSERVACIONES COMO HIDROMETEORÓLOGO}

Mis observaciones previas de temperatura del mar me ayudaron a encontrar el lugar más idóneo para medirla, teniendo en cuenta dos cosas fundamentales: las diferentes temperaturas del agua según la profundidad (en verano, sobre todo) y la barca de que disponía y que era la que utilizaban mi padre y mi abuelo para ir a pescar (Fig. 1), de poco más de 5 metros de eslora y que aún sigo usando para mi trabajo como hidrometeorólogo, aunque -en realidad, cuando me preguntan- yo digo que soy observador aficionado meteorológico y oceanográfico. El lugar donde hoy día sigo tomando la temperatura del agua y cuya profundidad alcanza los 90 metros, está situado a poco más de una milla de las Illes Medes y a unos $4 \mathrm{~km}$ del puerto de l'Estartit, en Girona.

Paralelamente, he ido tomando sistemáticamente (algunos desde finales de $1968 \mathrm{y}$ otros los he ido incorporando más tarde) distintos datos meteorológicos, como los de pluviometría, temperatura, humedad, evaporación, presión atmosférica, etc., datos que, gracias a la cesión de más aparatos por parte de la AEMET, se han ido incrementando con el paso de los años (por ejemplo, gracias a la instalación de un pluviómetro y de una estación automática; Tabla 1).

Mención especial merece la construcción de un mareógrafo casero (Fig. 3). A finales de los años ' 80 , en la televisión catalana (TV3) había un programa de divulgación y entretenimiento donde participaban institutos, escuelas de formación profesional, etc., en el que participó el instituto de bachillerato de Torroella de Montgrí (municipio al que pertenece l'Estartit). De acuerdo con el tema del programa, los centros educativos debían construir algún artilugio científico. Como no tenían una idea clara en este momento, el director del Instituto (Josep Pujol, que era y es amigo mío) me pidió que le diera alguna. Yo le dije que tenía en mente construir un mareógrafo algún día. Le gustó la cosa y me pidió un croquis. 


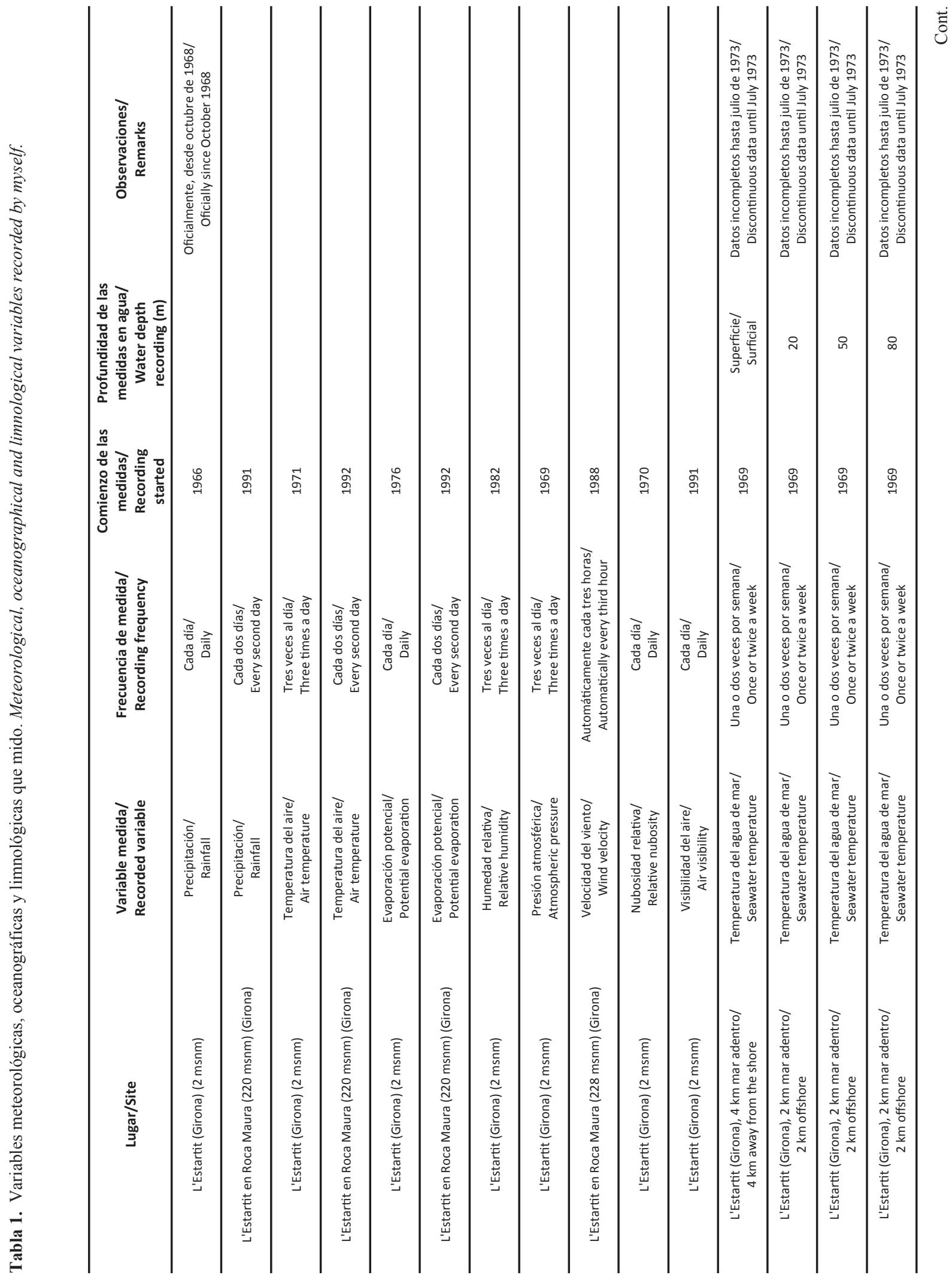




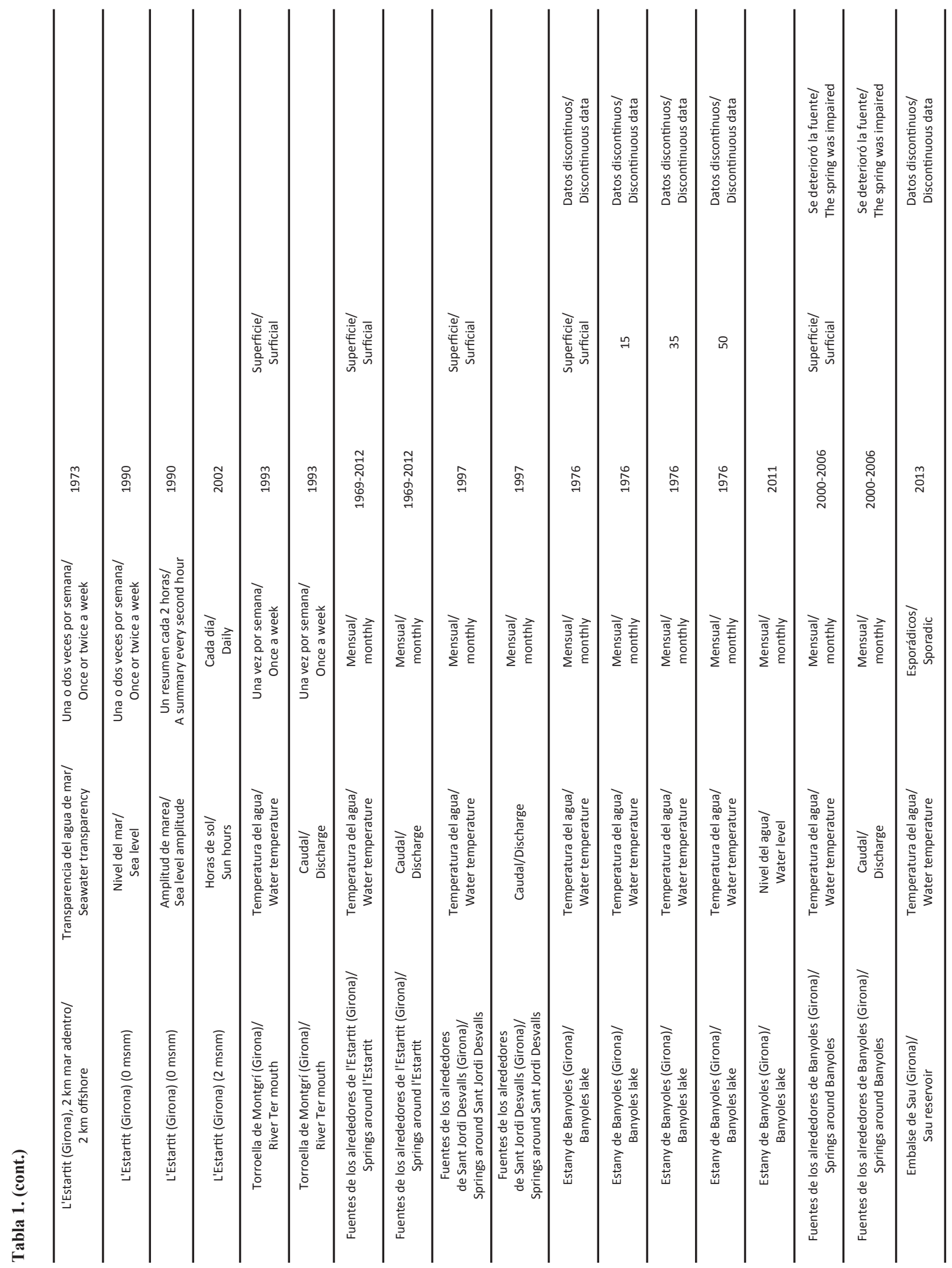


Alumnos y profesores construyeron el aparato que se instaló en el puerto de l'Estartit en enero de 1990, donde todavía sigue funcionando (Fig. 3). Gracias a él, ahora ya tengo casi 30 años de datos de marea, los cuales han servido para conocer el nivel medio del mar y -aparte de averiguar los ciclos de marea de la zona- nos permiten saber la tendencia y la variabilidad en el Mediterráneo occidental de este aspecto tan importante para detectar los efectos del cambio global.

Volviendo a la temperatura del mar y gracias a la sugerencia de un amigo biólogo (el Dr. Mikel Zabala), durante los años '90 solicitamos una subvención a la Generalitat para comprar una sonda CTD que me permitiera obtener datos verticales continuos de temperatura y salinidad (básicamente) durante cada muestreo. Y se nos concedió. En algún momento se estropeó (la electrónica y la sal no son muy buenos amigos) y luego el Instituto de Ciencias Marinas me cedió otra en 2012 que todavía sigue funcionando correctamente. Esto me permite detallar mejor las variables, si bien en algunos niveles marinos sigo midiendo también con los termómetros, para ir controlando la calibración de dicha sonda CTD.

A pocos kilómetros de mi zona de medidas marinas desagua el río Ter, lo cual afecta a la salinidad del mar en las áreas cercanas. Por eso, desde 1993 tomo también nota del caudal y de la
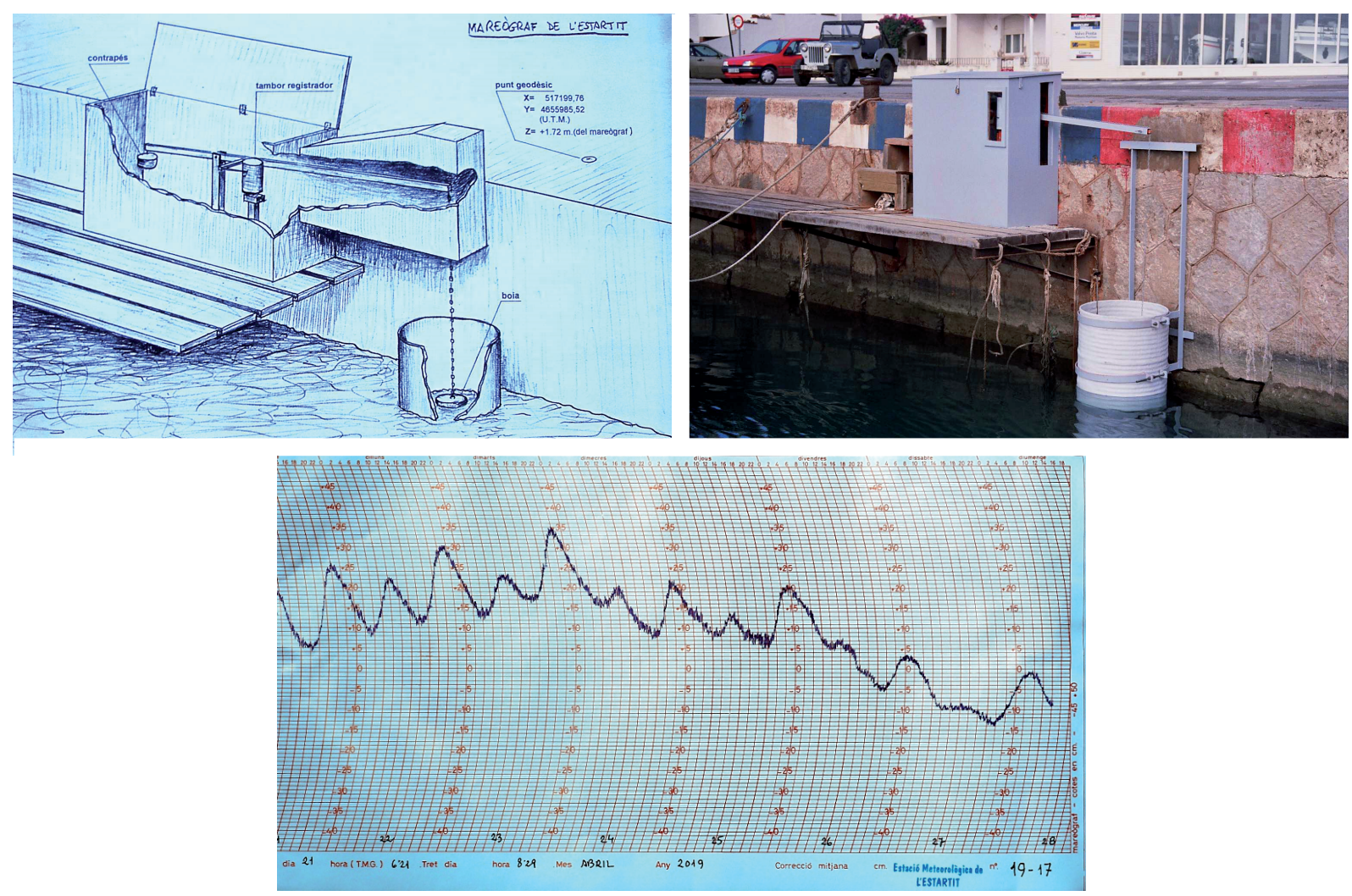

Figura 3. El mareógrafo diseñado por mí. Registra el nivel del mar en el puerto de l'Estartit (Girona) desde 1990 hasta la actualidad. A causa de unas obras se tuvo que instalar en otro lugar del puerto en 2008; sin embargo, técnicos del Institut Cartogràfic de Catalunya realizaron una nivelación de precisión desde el primer emplazamiento al segundo para conservar el mismo cero. Al lado del mareógrafo hay un clavo de referencia de nivelación para poder comprobar el "cero". En la imagen inferior se muestra un ejemplo reciente de los registros. The mareograph designed by myself, which has been recording sea levels from 1990 until nowadays in l'Estartit (Girona, NE Spain) bay. Because of some constructions the mareograph had to be moved to other site in 2008. However, technicians of the Catalonian Cartographic Institute undertook a precission leveling from one site to the other to preserve the same "zero". There is a reference notch beside the mareograph to check that zero. The lower picture shows an example of recent recordings. 
temperatura del agua de ese río en las cercanías de su desembocadura. Lo hago una vez por semana, aunque en caso de avenidas, más a menudo.

Aparte y aprovechando todo el material de medida de que dispongo, he observado con menor frecuencia la temperatura del lago de Banyoles (a unos $45 \mathrm{~km}$ ) y también alguna vez la he medido en algún embalse (como en Sau, Barcelona), con objeto de comparar su comportamiento térmico con el marino. Otros datos que tomo aproximadamente una vez al mes son la temperatura y el caudal de algunas fuentes cercanas a L'Estartit, los cuales de alguna manera indican el estrés hídrico del suelo.

En 2008, Carles Pelejero, del Instituto de Ciencias del Mar de Barcelona, me propuso colaborar para que, aprovechando mis salidas costeras, vinieran conmigo unos compañeros suyos una vez al mes para tomar unas muestras de agua y analizar el $\mathrm{pH}$ del agua. El motivo de realizar estas medidas es que se está observando que, con el aumento del $\mathrm{CO}_{2}$ atmosférico a causa de las actividades humanas, el mar absorbe parte de dicho compuesto y eso afecta al $\mathrm{pH}$ del agua, de forma que tiene lugar un aumento de la acidez. Esto puede afectar a todos los organismos que tienen alguna composición caliza, como -por ejemplo- los mejillones, las langostas y los corales, entre otros.

La medida de todas las variables que registro requiere bastante dedicación y, sobre todo, el ser metódico. Sin embargo, aunque vaya disponiendo de más aparatos de medida automáticos, aún hay bastantes variables que son fruto de la observación visual, como el estado del cielo, el tipo de nubes, la visibilidad del aire, etc. y, pese a que haya boyas de medida de oleaje, no siempre nos dicen si la mar más importante es la de viento o la de fondo, si hay mar cruzada, etc., lo cual todavía hace útil la observación cualitativa.

Entre las cosas menos agradecidas de mi trabajo como hidrometeorólogo están la calibración de los aparatos, para que los datos obtenidos sean comparables, y especialmente el tema de las averías o los problemas de funcionamiento que a veces suceden. Para la calibración de los termómetros de inversión utilizados en la observación de la temperatura del mar y que vienen de la casa constructora con una tabla de corrección para cada termómetro, los pongo en un cubo de agua junto al termómetro que quiero recalibrar $\mathrm{y}$, mezclando el agua, voy anotando la temperatura de unos y otros. Repito esto para diferentes temperaturas; en invierno, para temperaturas más bajas y en verano, para las más altas, a fin de evitar al máximo la interferencia de la temperatura ambiente. En el caso del evaporímetro "Piché", que uso para medir la evaporación, tomo el diámetro interior del tubo, la distancia entre las líneas de graduación y la superficie del papel secante que se le pone. Otro ejemplo similar consiste en relacionar la graduación y el diámetro de la probeta del pluviómetro con el diámetro de la boca del mismo pluviómetro. En los casos de avería o mal funcionamiento de los equipos conviene la reparación o sustitución del aparato con problemas para evitar al máximo las lagunas en las series de datos.

Todo junto requiere mucha dedicación, sin olvidar de hacer los resúmenes en soporte digital para que los estudiosos del clima, de la biología o de la fenología local puedan utilizar los datos sin destinar un esfuerzo mayor del que ya dedican a sus estudios específicos.

En todo, he recibido ayudas que, para quien las ofrece son bastante grandes, pero para quien las recibe, bastante escasas si tenemos en cuenta el tiempo de dedicación a todas las muchas tareas que he descrito. En mi caso, ese tiempo ha ido en aumento a lo largo de los años a medida que tenía más aparatos y más variables a observar, pero también debido a la realización de más observaciones directas sin aparatos, como las que hago del estado del mar de manera visual un par de veces al día: la altura de olas, su dirección, si es mar de fondo o mar de viento y, cuando se puede, si el oleaje es importante y/o las olas son bastante regulares, así como una estimación de la distancia entre crestas de dos olas consecutivas y el período del oleaje.

Durante los primeros años, gracias al Dr. Ballester publiqué algún artículo en la revista Inmersión y Ciencia (Pascual, 1971b), editado por la Federación Española de Actividades Subacuáticas, donde reseñaba algunos aspectos de mis observaciones marinas a cambio de una modesta retribución. Más tarde, colaboré en la monografía que sobre las Islas Medes publicó la Institució 
Catalana d'Historia Natural (Pascual \& Flos, 1984; Pascual et al., 1984) y amplié esos resultados preliminares en un trabajo posterior sobre el clima y el régimen térmico de las aguas de dichas islas y de la región del Montgrí (Pascual et al., 2012).

También he publicado varios artículos en revistas locales, como el "Llibre de la Festa Major de Torroella de Montgrí" o el "Llibre de la Festa de Santa Llúcia de l'Estartit", comentando episodios meteorológicos acaecidos en el nordeste de la Península Ibérica (Pascual, 2005, 2015, 2016). Finalmente, he colaborado en varios trabajos sobre la hidrología y la conservación de humedales del litoral catalán (Menció et al., 2017; Quintana et al., 2018).

A lo largo de unos varios años de la década de 1980 tuve ayudas de la Diputación de Girona, a cambio de proporcionarles un resumen de mis observaciones anuales. Finalmente, esa partida se trasvasó al espacio natural protegido que se creó: el Parc Natural del Montgrí, les Illes Medes i el Baix Ter (anteriormente la zona de las Illes Medes ya era Reserva Marina), y ahora recibo una ayuda económica de dicho Parque.

No debo olvidar la ayuda que representan, desde la AEMET, el material de observación meteorológica y, desde el ICM-CSIC, los aparatos de observación marina. Tampoco quiero dejar sin mencionar las facilidades prestadas por el Ayuntamiento local, Ports de la Generalitat y el Club Náutico de l'Estartit, para el mantenimiento de las zonas de instalación, ya que algunos de los aparatos están situados en lugares de su competencia. Con la AEMET, colaboro pasándoles mensualmente resúmenes de mis datos. En el caso del Instituto de Ciencias del Mar, también les aporto datos y resúmenes básicamente marinos. Al ayuntamiento de l'Estartit - a la Oficina de Turismo- le facilitó datos diarios para que puedan informar a quienes acudan allí y se interesen por ellos. Desde el Club Náutico o de los Ports de la Generalitat, a veces me solicitan datos referentes a algún acontecimiento adverso, como algún temporal de mar o viento fuerte y se los doy con gusto.

Por mis actividades de tantísimos años, la AEMET tuvo a bien darme un premio en 2018. Fue durante el Día Meteorológico Mundial, en el mes de marzo. En octubre de 2018 se cumplían 50 años de mi primera observación pluviométri- ca. Me invitaron a visitar las instalaciones centrales de la AEMET en Madrid, donde se encuentran las diversas secciones de previsión meteorológica, así como a las del superordenador para el almacenamiento de datos climáticos y la confección de modelos numéricos de previsión. Todo me pareció muy interesante. Coincidí allí con otros dos colaboradores de la AEMET (Miguel Ángel García Pérez, de O Carballiño, en Ourense, y Luís Manuel Sanz Rodríguez, de Quintanilla de Ricuerda, en Burgos), quienes también llevan bastantes años de observación meteorológica a sus espaldas. Tuvimos ocasión de intercambiar experiencias.

\section{RESULTADOS}

\section{El presente: algunas observaciones a largo plazo}

Mis datos hidrometeorológicos se almacenan en la "web" http://meteolestartit.cat. Evidentemente, los registros deben ser analizados por personas estudiosas de la Meteorología, de la Oceanografía y de la Limnología. Sin embargo, aunque sea un análisis sencillo, creo que los datos obtenidos evidencian una influencia de la actividad humana en el clima y en las aguas.

El hecho de que en l'Estartit la marea astronómica sea de poca amplitud (entre los 10 y los $25 \mathrm{~cm}$, según la fase de la luna), permite apreciar bien la tendencia del nivel del mar durante mis casi treinta años de observaciones y toma de datos (Fig. 4). El resultado es que el aumento medio del nivel del mar en esta zona se acerca a los $4 \mathrm{~mm}$ por año, presumiblemente a causa del deshielo de zonas polares y glaciares de montaña y de la dilatación del mar causada por el aumento de su temperatura.

Otros hechos que he observado son el aumento de la temperatura del aire, que ya supera un grado durante los últimos 45 años, y el de la temperatura del mar (Fig. 5), que es del orden de $1^{\circ} \mathrm{C}$ en la superficie y $0.5^{\circ} \mathrm{C}$ a unos 80 metros de profundidad, habiendo aumentado también la temperatura entre la superficie y esos 80 metros en valores intermedios en el rango $0.5-1{ }^{\circ} \mathrm{C}$. También vale la pena resaltar aquí, no tanto la tendencia de la pluviosidad (al ser la lluvia muy irregu- 


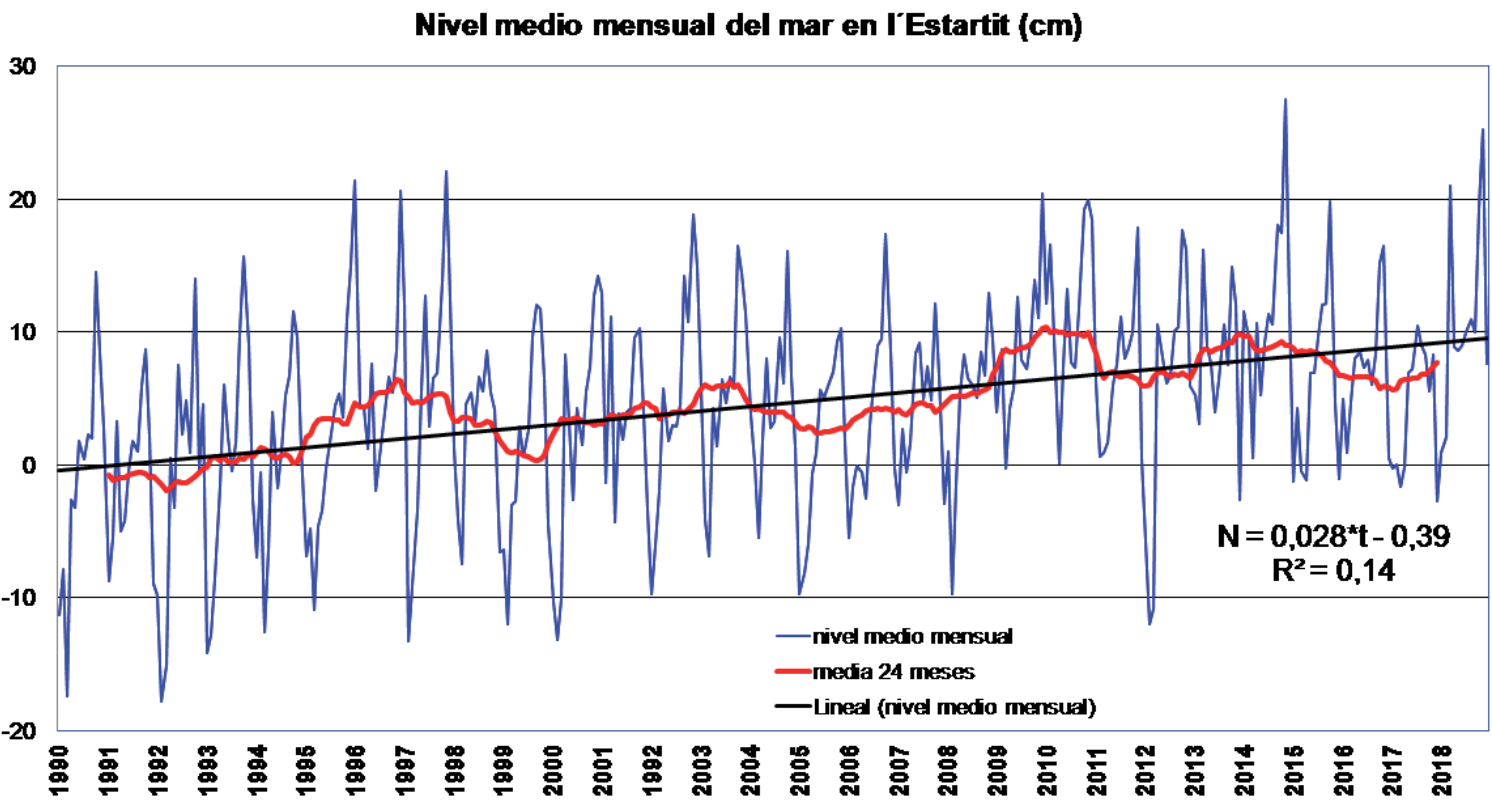

Figura 4. El nivel del mar en l'Estartit (Girona) desde 1990 hasta la actualidad. En rojo, la media móvil de 24 meses; en negro, la tendencia interanual. Sea level at l'Estartit (Girona, NE Spain) since 1990. While the red line is a moving average of 24 months, the black line is the long-term trend.

L'Estartit - Temperaturas medias del aire y del agua del mar $\left({ }^{\circ} \mathrm{C}\right)$

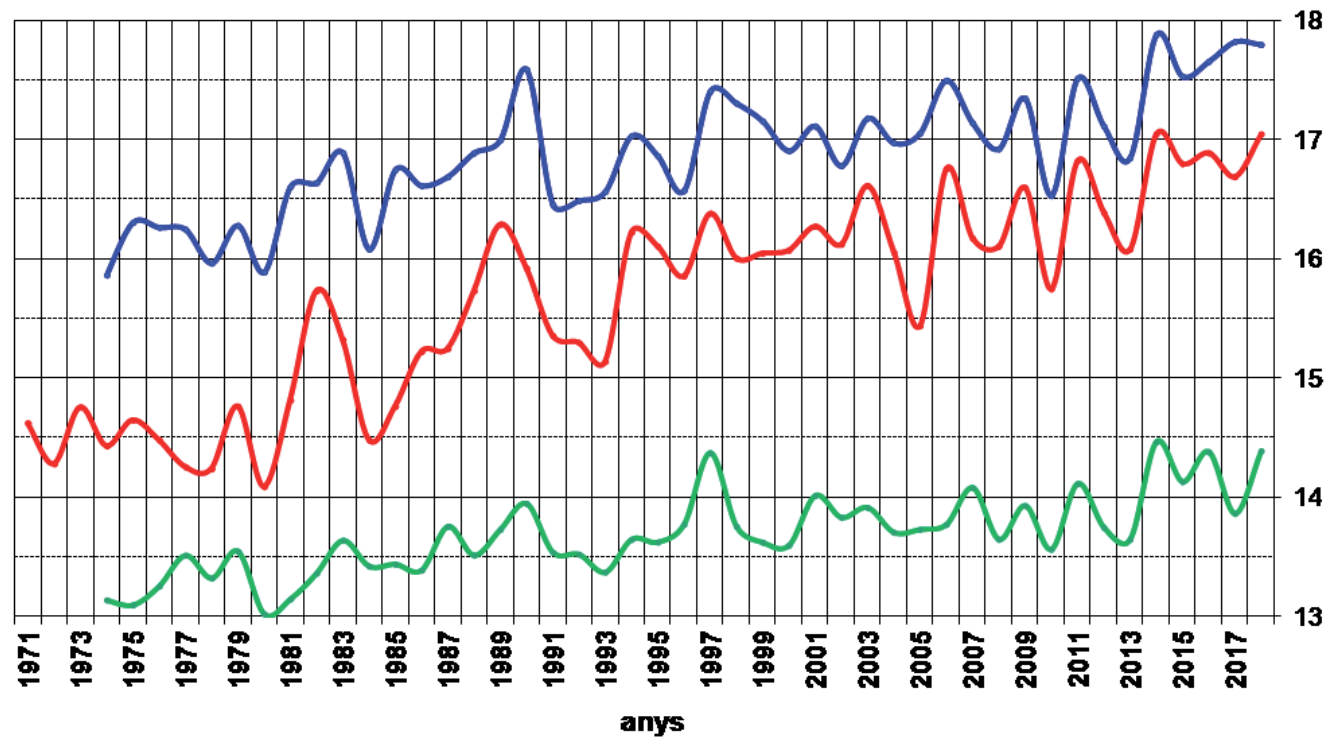

-temp. miljanes de laje -temp. mar en superficie -temp. mar $-80 \mathrm{~m}$.

Figura 5. Temperaturas atmosférica en el puerto de l'Estartit (desde 1971, en rojo) y del agua de mar en superfície (en azul) y a 80 de profundidad (desde 1974, en verde), estas últimas registradas $4 \mathrm{~km}$ mar adentro de l'Estartit. Promedios mensuales. Air temperature (red line) at l'Estartit bay since 1971 and surface (blue line) and $80 \mathrm{~m}$ (green line), monthly seawater temperatures recorded $4 \mathrm{~km}$ away from l'Estartit (NE Spain) since 1974. 
lar, resulta difícil de cuantificar), pero sí su mayor variabilidad: llueve de manera más intensa, pero hay períodos más largos de tiempo sin lluvia. Otra cosa también significativa que he observado es la tendencia al aumento la evaporación potencial en l'Estartit (Fig. 6).

Hay otras series públicas donde se registra el nivel del mar de la Península Ibérica: la mayoría están almacenadas en una base de datos del Reino Unido (https://www.psmsl.org/data/obtaining/ map.html). En dicha hoja "web" se puede ver que existen bastantes estaciones de observación en nuestras costas, pero la mayoría o han dejado de registrarse, o comenzaron a acumular datos después de que lo hiciese yo. Es notable la serie de Almería, que empezó a medirse en 1977, si bien lo ha sido en dos lugares diferentes. Lo más complicado de todo este asunto es que no haya lagunas de datos y que la serie conserve el mismo cero a lo largo de los años.

En cuanto a las medidas de temperatura marina, que yo sepa, en el Centre d'Estudis Avançats de Blanes (CSIC), desde 2009 a 2018 se han hecho observaciones quincenales con sonda
CTD, delante del cañón submarino de Blanes hasta un fondo de 220 metros. También en ese sitio hubo una boya que efectuaba registros térmicos continuos en la zona más cercana a la superficie, pero se perdió a causa de un temporal hace un par de años. Más o menos, también desde 2008 se llevan realizando unas observaciones mensuales delante de la costa de Barcelona, como complemento del muestreo para el estudio de la acidificación del mar; lo hace el equipo de Carles Pelejero, del Instituto de Ciencias del Mar (CSIC). Además, en Barcelona, un observador aficionado (Diego Lázaro) realiza observaciones semanales de la temperatura superficial del mar en la playa de la Barceloneta desde 2008. Finalmente, cabe señalar también que las boyas de "Puertos del Estado" registran medidas de temperatura en distintos puertos.

Mis observaciones limnológicas comenzaron más tarde y las centro en los estudios de la desembocadura del río Ter, en el estany de Banyoles y en algunas fuentes del entorno, donde mido la temperatura del agua, el caudal, el nivel lacustre, etc. (Tabla 1). A diferencia de lo que

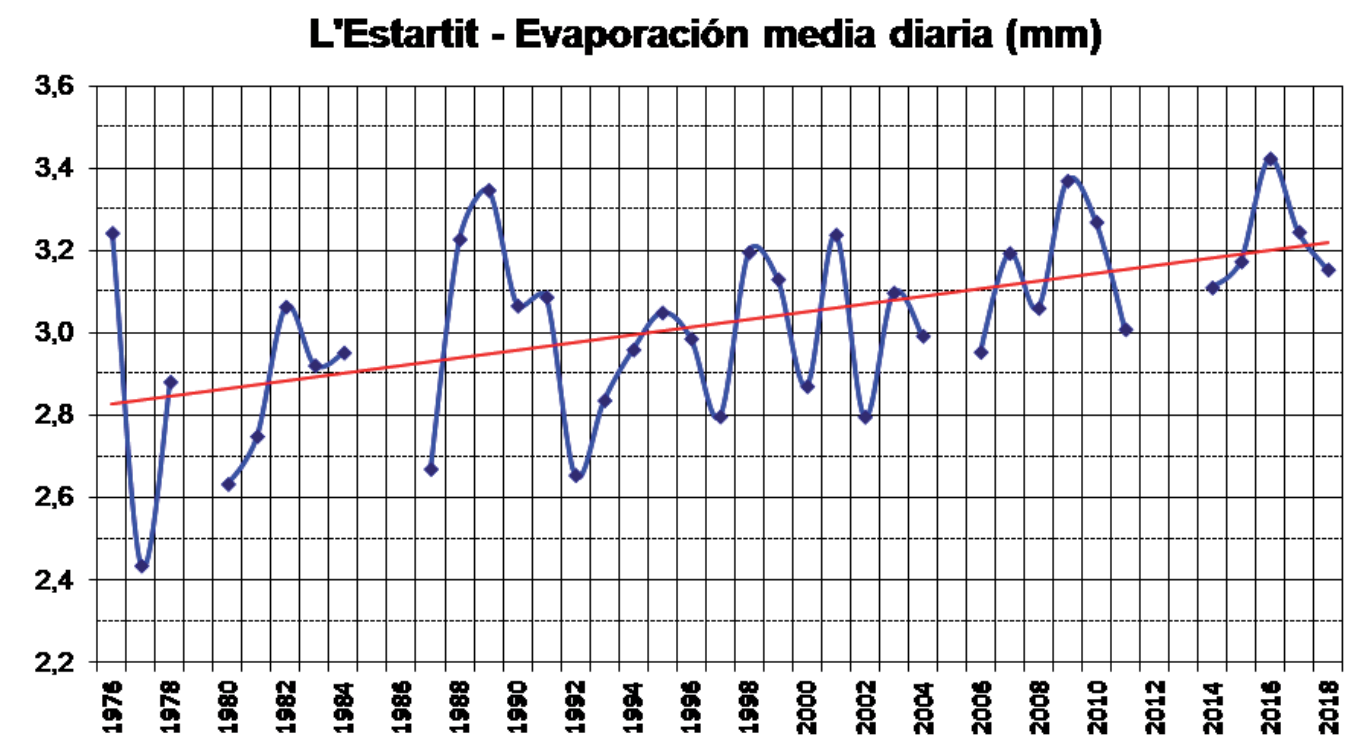

Figura 6. Evaporación potencial mensual, con datos medios diarios, en l'Estartit (Girona), medida mensualmented
potential evaporation at l'Estartit (Girona, NE Spain), recorded since 1976. The red line is the long-term trend. 
sucede en el mar (Figs. 4-5), ni en el Ter ni en Banyoles he detectado aún tendencia alguna (Figs. 7-9), quizá porque la longitud de los registros todavía sea insuficiente.

\section{El futuro de mis observaciones hidrometeoro- lógicas}

Yo, como muchos observadores meteorológicos, comencé por afición. He sentido cada vez más curiosidad por otras variables y he hecho toda clase de observaciones diversas. Lo más complicado de llevar a cabo es la regularidad en las observaciones. A veces, me vienen jóvenes interesados en cómo hago las observaciones, y les enseño los aparatos y la manera de hacerlo. A lo mejor, alguno de ellos continúa mi labor.

En todo caso, diversas instituciones (Instituto de Ciencias del Mar; Servei Meteorològic de Catalunya; Parc Natural del Montgrí, Illes Medes i Baix Ter), también se han interesado para que, en un futuro, se pueda continuar este trabajo. Tengo esperanzas de que continuará, si no para todas las variables que ahora registro, como mínimo para las más útiles a nivel científico y de otras aplicaciones (pesca, turismo de buceo, etc.).

\section{CONCLUSIONES}

Por entender la mar y ayudar a mi familia y amigos pescadores, fui interesándome desde joven por la meteorología y por las observaciones térmicas de las aguas, tanto en el mar (primero) como en el agua dulce (después). Mi propia iniciativa y la de investigadores científicos reconocidos hicieron que poco a poco fuese registrando datos de esa índole desde finales de la década de 1960 hasta la actualidad. Dichas observaciones las almaceno en una hoja "web" de carácter público (http://meteolestartit.cat), donde quedan a disposición de cualquier persona o institución interesada. Creo que mi iniciativa privada en el campo de la hidrometeorología es una de las pocas existentes en España que tiene series tan largas de datos. Dichos datos pueden ser de utilidad no solo a corto plazo para actividades turísticas (navegación, buceo, etc.), pero también para las ciencias ambientales, pues permiten documentar tendencias y variabilidades meteorológicas, oceanográfi-

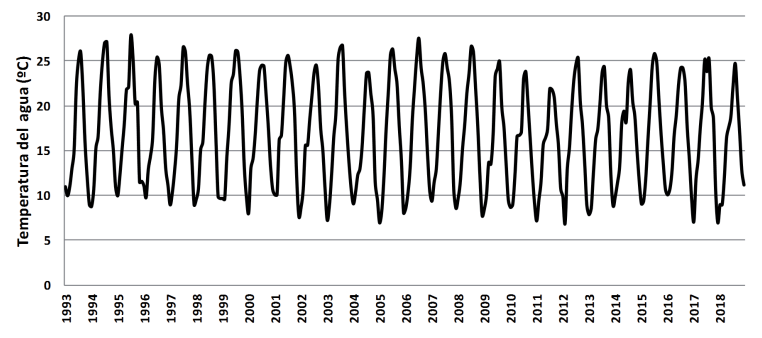

Figura 7. Temperatura del agua del río Ter a $5 \mathrm{~km}$. de su desembocadura (Girona), registrada desde 1993. Water temperature of Ter river recorded at its mouth in the sea (Girona, NE Spain) since 1993.

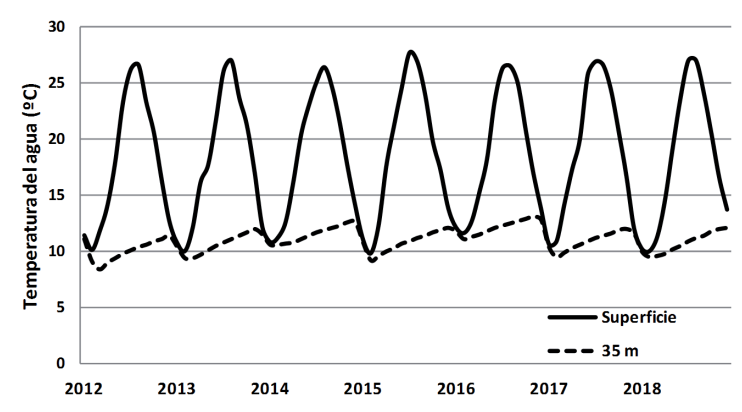

Figura 8. Temperatura del agua en el estany de Banyoles (Girona) a dos profundidades. Datos registrados en la cubeta I del plano de Ramon Margalef de 1969 (véase Moreno-Amich \& García-Berthou, 1989), situada enfrente del Club Natació Banyoles. Water temperatures of Banyoles lake (Girona, NE Spain) at two different depths, recorded in basin I of Ramón Margalef 1969 plot (see Moreno-Amich \& Garcia-Berthou, 1989), located in front of Banyoles Swimming Club.

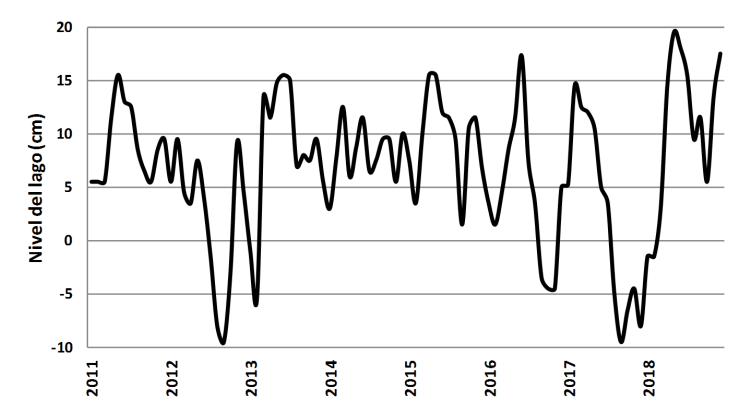

Figura 9. Nivel del agua en el estany de Banyoles (Girona) desde 2011 hasta la actualidad; el nivel 0 es el nivel Darder, nombre de Francesc Darder Llimona, veterinario y pionero de los estudios sobre Banyoles y su lago. En la orilla del mismo colocó una señal en el mismo lugar donde años después se instaló una regla de medida del nivel que aún se conserva y se conoce como nivel cero del lago. Changes in water level of Banyoles lake (Girona, NE Spain) since 2011. Level 0 is called the Darder level, which was named as Francesc Darder Llimona, a veterinarian and a pioneer of studies on Banyoles area and its lake. He set up a sign post at the same site where a still-existing level was installed long after and it is now known as the zero lake level. 
cas y limnológicas en el nordeste de la Península Ibérica y ayudar a la caracterización del cambio global que ocurra en nuestros territorios.

\section{AGRADECIMIENTOS}

Las instituciones que me han ayudado a lo largo de tantos años y a las que estoy muy agradecido son las siguientes: la AEMET; el ayuntamiento de Torroella-l'Estartit (Girona); el Club Nàutic Estartit; el Institut de Ciències del Mar de Barcelona; el Parc Natural del Montgrí, les Illes Medes i Baix Ter; y Ports de la Generalitat.

\section{BIBLIOGRAFÍA}

MENCIÓ, A., X. CASAMITJANA, J. MAS-PLA, N. COLL, J. COMPTE, M. MARTINOY, J. PASCUAL \& X. QUINTANA. 2017. Groundwater dependence of coastal lagoons: The case of La Pletera salt marshes (NE Catalonia). Journal of Hydrology, 552: 793-806. DOI: 10.1016/j.jhydrol.2017.07.034.

MORENO-AMICH, R. \& E. GARCÍA-BERTHOU. 1989. A new bathymetric map based on echo-sounding and morphometrical characterization of the Lake of Banyoles (NE-Spain). Hydrobiologia, 185: 83-90.

PASCUAL, J. 1971a. Geografía de la región de las Islas Medas. Inmersión y Ciencia, 3: 7-33.

PASCUAL, J. 1971b. Aproximación al estudio de las mareas en el Estartit. Inmersión y Ciencia, 3: 55-62.

PASCUAL, J. 2005. Les esllavissades de 1994 a l'Estartit. Revista La Punxa, 39: 26-37.

PASCUAL, J. 2015. Moviments de la platja durant les darreres dues dècades. Llibre de la
Festa Major de Torroella de Montgri: 147-158.

PASCUAL, J. 2016. Mareògraf de l'Estartit, més d'un quart de segle de dades. Penell, 44: $18-22$.

PASCUAL, J. \& J. FLOS. 1984. Meteorologia i oceanografia. In: El sistemes naturals de les Illes Medes (J. D. ROS, I. OLIVELLA \& J. M. GILI, eds.): 75-114. Institució Catalana d'Historia Natural. Arxius de la Secció de Ciències, 73. Barcelona.

PASCUAL, J., M. ZABALA, I. OLIVELLA \& J. M. GILI. 1984. Mapa batimètric, topogràfic i toponimic. In: El sistemes naturals de les Illes Medes (J.D. ROS, I. OLIVELLA \& J.M. GILI, eds.). Institució Catalana d'Historia Natural. Arxius de la Secció de Ciències, 73. Barcelona.

PASCUAL, J., N. BENSOUSSAN, J. SALAT \& J. GARRABOU. 2012. Clima i règim tèrmic de les aigües de les Illes Medes i el Montgrí. Recerca i Territori, 4: 65-77.

QUINTANA, X., D. BOIX, X. CASAMITJANA, A. COLOMER, J. COMPTE, D. CUNILLERA-MONTCUSÍ, S. GASCÓN, F. GICH, A. MENCIÓ, M. MARTINOY, J. MONTANER, J. PASCUAL, J. SALA, J. SOLÀ \& I. TORNERO. 2018. Management and restoration actions of confined Mediterranean coastal lagoons in the Baix Empordà and Baix Ter wetlands. In: Management and restoration of Mediterranean coastal lagoons in Europe (X. Quintana, D. Boix, S. Gascón \& J. Sala, eds.): 173-192. Recerca i Territori, 10. Càtedra d'Ecosistemes Litorals Mediterranis Parc Natural del Montgrí, les Illes Medes i el Baix Ter Museu de la Mediterrània. Girona. 